\title{
Stochastic Cost-Optimization and Risk Assessment of in situ Chemical Oxidation for Dense Non-Aqueous Phase Liquid (DNAPL) Source Remediation
}

\author{
Ungtae Kim \\ Cleveland State University, u.kim@csuohio.edu \\ Jack C. Parker \\ University of Tennessee, Knoxville \\ Robert C. Borden \\ North Carolina State University
}

Follow this and additional works at: https://engagedscholarship.csuohio.edu/encee_facpub

Part of the Environmental Engineering Commons

How does access to this work benefit you? Let us know!

Publisher's Statement

https://link.springer.com/article/10.1007/s00477-018-1633-y

\section{Recommended Citation}

Kim, Ungtae; Parker, Jack C.; and Borden, Robert C., "Stochastic Cost-Optimization and Risk Assessment of in situ Chemical Oxidation for Dense Non-Aqueous Phase Liquid (DNAPL) Source Remediation" (2019). Civil and Environmental Engineering Faculty Publications. 133.

https://engagedscholarship.csuohio.edu/encee_facpub/133

This Article is brought to you for free and open access by the Civil and Environmental Engineering at EngagedScholarship@CSU. It has been accepted for inclusion in Civil and Environmental Engineering Faculty Publications by an authorized administrator of EngagedScholarship@CSU. For more information, please contact library.es@csuohio.edu. 


\title{
Stochastic cost-optimization and risk assessment of in situ chemical oxidation for dense non-aqueous phase liquid (DNAPL) source remediation
}

\author{
Ungtae Kim Jack C. Parker · Robert C. Borden
}

\begin{abstract}
This study involved development of a computer program to determine optimal design variables for in situ chemical oxidation (ISCO) of dense nonaqueous phase liquid (DNAPL) sites to meet site-wide remediation objectives with minimum life-cycle remediation cost while taking uncertainty in site characterization data and model predictions into consideration. A physically-based ISCO performance model computes field-scale DNAPL dissolution, instantaneous reaction of oxidant with contaminant and with readily oxidizable natural oxidant demand (NOD), second-order kinetic reactions for slowly oxidizable NOD, and time to reach ISCO termination criteria. Remediation cost is computed by coupling the performance model with a cost module. ISCO termination protocols are implemented that allow different treatment subregions (e.g., zones with different estimated contaminant concentrations) to be terminated independently based on statistical criteria related to confidence limits of contaminant concentrations estimated from soil and/or groundwater sampling data. The ISCO model was implemented in the program called Stochastic Cost Optimization Toolkit, which includes modules for additional remediation technologies that can be implemented serially or in parallel coupled with a dissolved plume model to enable design optimization to meet plume-scale cleanup objectives. This study focuses on optimization of ISCO design to meet specified source zone remediation objectives. ISCO design parameters considered for optimization include oxidant concentration and injection rate, frequency and number of soil or groundwater samples, and cleanup criteria for termination of subregion injection. Sensitivity studies and example applications are presented to demonstrate the benefits of proposed stochastic optimization methodology.
\end{abstract}

Keywords Stochastic optimization · In situ chemical oxidation · Risk assessment · DNAPL source remediation · Uncertainty analysis

\section{Introduction}

A variety of technologies have been applied to remediate dense non-aqueous phase liquid (DNAPL) source areas over the last 40 years, including thermal source reduction, 
enhanced bioremediation, and in situ chemical oxidation (ISCO). Our focus here is on ISCO, which involves injection of a chemical reagent into the subsurface to oxidize contaminants. The technology has been studied since the early 1990s and has been used extensively in the field for a variety of contaminants (Huling and Pivetz 2006; McGuire et al. 2006; Siegrist et al. 2006, 2008; Borden et al. 2010; Krembs et al. 2010). Common reagents include potassium or sodium permanganate, persulfate, ozone, hydrogen peroxide $\left(\mathrm{H}_{2} \mathrm{O}_{2}\right)$, and modified Fenton's reagent $\left(\mathrm{H}_{2} \mathrm{O}_{2}\right.$ plus ferrous iron).

One of the most common oxidant delivery methods involves injecting pulses of oxidant solution into a network of injection wells, followed by periods in which oxidant reacts with contaminants and migrates with the natural groundwater flow. This approach is well adapted to the treatment of DNAPL source zones in moderate permeability aquifers (Crimi and Siegrist 2005; Siegrist et al. 2006, 2008; Borden et al. 2010; Cha and Borden 2012). In aquifers with a high groundwater velocity, low residence times in the target zone diminish the cost effectiveness of this method.

Remediation system design is to a great degree a problem of managing uncertainty. Although ISCO is a mature technology with well-documented design protocols, including available software for design and cost estimation (Borden et al. 2010; Siegrist et al. 2010), these tools do not address the effects of uncertainty in site characteristics or measurements used to make termination decisions on system performance and cost. Considerable work has been reported on optimization of long-term monitoring to trade off costs against the value of information (Loaiciga et al. 1992; U.S. EPA 2000, 2007; Reed et al. 2000). Compared to deterministic optimization, stochastic optimization can consider the prediction uncertainty by simulating a forward model for realistic ranges of parameters in decision-making (Freeze 2004; Bastante et al. 2008; Cardiff et al. 2010; Parker et al. 2011).

Stochastic optimization methods employ Monte Carlo simulations to define probability distributions of performance and cost for a given design and use optimization algorithms to determine design variables that minimize probability-weighted cost subject to performance constraints (Bastante et al. 2008; Cardiff et al. 2010; Parker et al. 2011; $\mathrm{Li}$ et al. 2015, 2017). Optimal remediation solutions may also be affected by economic and political uncertainty, such as changes in inflation, interest rates or regulations (Freeze 2004). These factors can, in principle, be addressed by Monte Carlo simulations. However, given the difficulty to quantify the associated probability distributions, a more practical approach may be to use sensitivity analyses to qualitatively factor their effect into the decision process, and to reoptimize design parameters periodically to consider evolving conditions. The purpose of the research reported here is to develop a decision protocol for ISCO design for incorporation into the Stochastic Cost Optimization Toolkit (SCOToolkit), which couples performance models for multiple remediation technologies with a dissolved transport module to identify the most cost-effective strategies to meet compliance criteria for sites with DNAPL sources (Parker et al. 2011; Kim et al. 2013; Lee et al. 2012).

\section{Methods}

\subsection{DNAPL source depletion during ISCO}

We consider a DNAPL source zone of bulk aquifer volume $V_{0}\left[\mathrm{~L}^{3}\right]$ to which we wish to apply ISCO. The total contaminant mass prior to ISCO is $M_{0}[\mathrm{M}]$ and the corresponding contaminant dissolution rate is $J_{0}\left[\mathrm{MT}^{-1}\right]$. We wish to consider potential efficiency improvements by dividing the source zone into smaller operational units that are monitored and managed independently. For example, field data may indicate one or more "hot spots" having high soil concentrations, other areas with moderate concentrations, and peripheral zones of lower concentrations. Since lower concentration zones will likely require fewer oxidant injections, earlier termination may be possible making less aggressive design variables more cost effective in these areas. Therefore, we consider dividing the source area into $N_{T Z}$ treatment zones (TZ) based on initial contaminant distributions within the source area. For each $\mathrm{TZ}_{i}$, the DNAPL source dissolution rate is described by a power function source depletion model as

$J_{i}(t)=F_{m t i}(t) F_{k i}(t) J_{0 i}\left(\frac{M_{i}(t)}{M_{0 i}}\right)^{\beta}$

where $J_{i}(t)$ is the dissolution rate from source zone for $\mathrm{TZ}_{i}$ as a function of time $\left[\mathrm{MT}^{-1}\right], M_{i}(t)$ is the DNAPL mass remaining at time $t[\mathrm{M}], M_{0 i}$ is the mass just prior to ISCO $[\mathrm{M}], J_{0 i}$ is the initial dissolution rate $\left[\mathrm{MT}^{-1}\right], \beta$ is a depletion exponent [-], $F_{m t ~} i$ is a mass transfer enhancement factor associated with high oxidant concentrations, which is 1 with no enhancement and $>1$ with increasing enhancement [-], and $F_{k i}$ is a mass transfer inhibition factor due to pore clogging that ranges from 1 with no pore clogging to 0 with complete clogging [-]. The power function DNAPL source model has been widely used and validated by high resolution numerical simulations, laboratory experiments and field studies (Rao and Jawitz 2003; Parker and Park 2004; Falta et al. 2005; Jawitz et al. 2005; Park and Parker 2005; Parker and Falta 2008; Cardiff et al. 2010; Parker et al. 2010a; Parker and Kim 2015; Yang et al. 2016). The depletion exponent $\beta$ depends on the 
DNAPL "architecture," i.e., the geometry within the pore space. Values $<1$ are typical of DNAPL pools or lenses, while values $>1$ reflect residual DNAPL with a more discontinuous structure (Parker and Park 2004).

The mass transfer enhancement factor, $F_{m t}$, may be estimated based on Reitsma and Dai (2001) as

$F_{m t}(t)=1+f_{m t} \frac{C_{o x}(t) W_{c t}}{S_{c t} W_{o x}}$

$f_{m t}=\frac{D_{o x}}{n_{o x / c t} D_{c t}}$

where $C_{o x}(t)$ is the current aqueous oxidant concentration $\left[\mathrm{ML}^{-3}\right], S_{c t}$ is the effective solubility of contaminant in the DNAPL $\left[\mathrm{ML}^{-3}\right], W_{o x}$ is the molecular weight of oxidant $\left[\mathrm{M} \mathrm{mol}^{-1}\right], W_{c t}$ is the molecular weight of contaminant $\left[\mathrm{M} \mathrm{mol}^{-1}\right], D_{o x}$ is the aqueous oxidant diffusion coefficient $\left[\mathrm{L}^{2} \mathrm{~T}^{-1}\right], D_{c t}$ is the aqueous contaminant diffusion coefficient $\left[\mathrm{L}^{2} \mathrm{~T}^{-1}\right]$, and $n_{o x / c t}$ is the molar ratio of oxidant to contaminant for the redox reaction.

If permanganate is used as the ISCO oxidant, pore clogging by $\mathrm{MnO}_{2}$ precipitation can inhibit DNAPL dissolution. This is modeled following West et al. (2007) and West and Kueper (2012) as

$F_{k}(t)=1-\left|S_{\text {rind }}\right| C_{\mathrm{MnO}_{2}}(t)$

where $C_{\mathrm{MnO}_{2}}(t)$ is the mass of precipitated $\mathrm{MnO}_{2}$ per volume of water in the $\mathrm{TZ}\left[\mathrm{ML}^{-3}\right]$ as a function of time, and $S_{\text {rind }}$ is the slope of the relationship between relative permeability and $\mathrm{MnO}_{2}$ concentration.

Contaminant soil concentrations are assumed to be lognormally distributed, which has been shown to be a good approximation for DNAPL source zones by Parker et al. (2017). Therefore, initial mass $M_{0 i}$ and mass flux $J_{0 i}$ values in each TZ are generated stochastically using a log-normal distribution for Monte Carlo simulations from the estimated upper and lower confidence limits of soil concentrations $\left(C_{\text {soil }}^{\min }\right.$ and $\left.C_{\text {soil }}^{\max }\right)$ within each $\mathrm{TZ}$ with a bulk volume $V_{0 i}$ based on site characterization data. For example, using a log-normal distribution, the log mean of soil concentration is the median and the confidence limits is assumed as $95 \%(\approx \pm 2 \sigma)$ for practical applications. Now, we can generate a mass for $\mathrm{TZ}_{i}$ as $M_{0 i}=\exp \left\{0.5 \ln \left(C_{\text {soil }}^{\text {min }} C_{\text {soil }}^{\text {max }}\right)+\right.$ $\left.0.25 \ln \left(C_{\text {soil }}^{\max } / C_{\text {soil }}^{\min }\right) \mathrm{U}(0,1)\right\}$, where $\mathrm{U}(0,1)$ is a uniform random number. Finally, the generate mass and mass flux values are reconditioned to meet the mass conservation requirements

$\sum_{i=1}^{N_{T Z}} M_{0 i}=M_{0}, \sum_{i=1}^{N_{T Z}} J_{0 i}=J_{0}$, and $\sum_{i=1}^{N_{T Z}} V_{0 i}=V_{0}$.

\subsection{ISCO reaction model}

Within each TZ an injection well network is constructed to inject oxidant at a specified concentration and flow rate for a defined duration such that injection zones for each well overlap at the end of an injection period. Following the injection period, oxidant is assumed to migrate under natural gradient conditions until treatment is terminated or another injection event is undertaken. Since TZs consist of multiple overlapping injection zones with injection periods that are short compared to subsequent natural gradient periods, it is reasonable to approximate the oxidant concentration in the $\mathrm{TZ}$ as uniform at the end of the injection period and to treat the $\mathrm{TZ}$ as a stirred reactor. Mass balance equations are solved for the following components within each TZ.

- DNAPL contaminant

- Aqueous and adsorbed phase contaminant

- Rapidly oxidizable natural oxidant demand ("fast" NOD or $N O D_{f}$ )

- Slowly oxidizable natural oxidant demand ("slow" NOD or $N O D_{s}$ )

- Aqueous phase oxidant

DNAPL serves as a rate-limited source of aqueous contamination described by Eqs. (1)-(3). Direct oxidation of DNAPL contaminant is assumed to be negligible. However, DNAPL dissolution rate is coupled with aqueous oxidant concentration via Eq. (2). Following Cha and Borden (2012), oxidant is assumed to react instantaneously with aqueous and adsorbed contaminant and with $N O D_{f}$. Oxidation of $N O D_{s}$ is modeled as a second-order kinetic reaction. Equilibrium is assumed between aqueous phase and adsorbed contaminant.

NOD is characterized by the total NOD per dry soil mass $\left(C_{\text {soil }}^{\text {NODtot }}\right)$, the ratio of fast NOD to total NOD $\left(f_{\text {NODf }}\right)$, and the second order rate coefficient for NODs $\left(k_{N O D s}\right)$. NOD parameters may be determined on soil samples using the laboratory protocol described by ASTM method D7262-07 (ASTM 2007). Since potassium permanganate is the oxidant in this lab test, the quantity of NOD is typically reported as moles of $\mathrm{KMnO}_{4}$. Typical ranges for NOD parameters are given in Table 1 based on Cha (2012).

The reaction between a chlorinated solvent and an $\mathrm{O}_{2}-$ equivalent oxidant $\left(\mathrm{O}_{2 \text { eq }}\right)$ may be written generically as

$$
\mathrm{C}_{\mathrm{a}} \mathrm{Cl}_{\mathrm{b}} \mathrm{H}_{\mathrm{c}}+n_{\mathrm{O}_{2} e q / c t} \mathrm{O}_{2 \mathrm{eq}} \rightarrow n_{\mathrm{CO}_{2}} \mathrm{CO}_{2}+n_{\mathrm{Cl}_{2}} \mathrm{Cl}_{2}+n_{\mathrm{H}_{2} \mathrm{O}} \mathrm{H}_{2} \mathrm{O}
$$

with stoichiometric coefficients $n_{O_{2} e q / c t}=\mathrm{a}+\mathrm{c} / 4$, $n_{\mathrm{CO}_{2}}=\mathrm{a}, n_{\mathrm{Cl}_{2}}=\mathrm{b} / 2$, and $n_{\mathrm{H}_{2} \mathrm{O}}=\mathrm{c} / 2$. For common chlorinated solvents, $\mathrm{O}_{2}$-equivalent oxidant requirements for complete oxidation of one mole of contaminant $\left(n_{\mathrm{O}_{2} e q / c t}\right)$ 
Table 1 Typical natural oxygen demand (NOD) parameter ranges (Cha 2012)

\begin{tabular}{lllll}
\hline Parameter & $10 \% \mathrm{LCL}^{\mathrm{a}}$ & Median & $90 \% \mathrm{UCL}^{\mathrm{a}}$ & $\mathrm{Unit}^{\mathrm{b}}$ \\
\hline Total NOD per soil mass $\left(C_{\text {soil }}^{\text {NODtot }}\right)$ & 2 & 28 & 158 & $\mathrm{mmol} / \mathrm{kg}$ \\
Fraction of "fast" NOD $\left(f_{\text {NODf }}\right)$ & 0.028 & 0.126 & 0.361 & - \\
"Slow" NOD rate constant $\left(k_{\text {NODs }}\right)$ & 0.003 & 0.018 & 0.395 & $\mathrm{~L} / \mathrm{mmol}$ day \\
\hline
\end{tabular}

${ }^{a} \mathrm{LCL}$ and UCL $=$ Lower and Upper Confidence Limit, respectively

${ }^{\mathrm{b}}$ Reported as mmol of $\mathrm{MnO}_{4}^{-1}$ (to obtain $\mathrm{mmol} \mathrm{O}_{2}$ eq multiply by $n_{O_{2} e q / o x}=0.75$ ) are thus 1 for $\mathrm{CCl}_{4}$ (carbon tetrachloride, CT), 2 for $\mathrm{C}_{2} \mathrm{Cl}_{4}$ (perchloroethylene, PCE), 2.25 for $\mathrm{C}_{2} \mathrm{HCl}_{3}$ (trichloroethylene, TCE), 2.5 for $\mathrm{C}_{2} \mathrm{H}_{2} \mathrm{Cl}_{2}$ (dichlororethylene, DCE), and 2.75 for $\mathrm{C}_{2} \mathrm{H}_{3} \mathrm{Cl}$ (vinyl chloride, VC). Oxidation reactions for other contaminants may be written in a similar manner to determine $n_{O_{2} e q / c t}$.

The net stoichiometry for contaminant-oxidant pairs ( $c t$, $o x$ ) may be computed as

$n_{o x / c t}=\frac{n_{O_{2} e q / c t}}{n_{O_{2} e q / o x}}$

where $n_{o x / c t}$ is the molar ratio of actual oxidant utilized to contaminant for a given ct-ox pair, $n_{\mathrm{O}_{2} e q / c t}$ is the $\mathrm{O}_{2^{-}}$ equivalent moles of oxidant per mole of the contaminant of interest (Eq. 5), and $n_{\mathrm{O}_{2} e q / o x}$ is the $\mathrm{O}_{2}$-equivalent moles of the oxidant. For common ISCO oxidants, $n_{\mathrm{O}_{2} e q / o x}$ is approximately 0.75 for permanganate $\left(\mathrm{MnO}_{4}{ }^{-}\right), 1.5$ for ozone $\left(\mathrm{O}_{3}\right)$, and 2 for hydrogen peroxide $\left(\mathrm{H}_{2} \mathrm{O}_{2}\right)$ or Fenton's reagent, although values can vary due to reaction complexity. Approximate net reaction stoichiometries for various contaminants and oxidants computed from Eq. (6) are summarized in Table 2.

Mass balance equations for DNAPL contaminant, dissolved and adsorbed contaminant, oxidant, $N O D_{f}$ and $N O D_{s}$ are solved for each TZ over time based on the sequential modeling approach proposed by Cha and Borden (2012) as follows:

(1) Compute quantity of contaminant released from DNAPL due to dissolution over the current time

Table 2 Estimated reaction stoichiometries for various contaminants and oxidants

\begin{tabular}{lllll}
\hline Contaminant & $\mathrm{O}_{2}$ equivalent & \multicolumn{3}{c}{ Oxidant utilization ratio $\left(n_{\text {ox } / c t}\right)$} \\
\cline { 3 - 5 } & $n_{O_{2} \text { eq } / c t}$ & $\mathrm{MnO}_{4}$ & Ozone & $\mathrm{H}_{2} \mathrm{O}_{2}$ \\
\hline $\mathrm{CT}$ & 1.00 & 1.33 & 0.67 & 0.50 \\
$\mathrm{PCE}$ & 2.00 & 2.67 & 1.33 & 1.00 \\
$\mathrm{TCE}$ & 3.24 & 4.32 & 2.16 & 1.62 \\
$\mathrm{DCE}$ & 2.50 & 3.33 & 1.67 & 1.25 \\
$\mathrm{VC}$ & 2.75 & 3.67 & 1.83 & 1.38 \\
\hline
\end{tabular}

step. Update DNAPL mass remaining and dissolved plus adsorbed contaminant mass.

(2) Solve mass balance for oxidant reactions with aqueous and adsorbed contaminant.

(3) Compute oxidant loss by downgradient outflow from $\mathrm{TZ}$ and dilution by upgradient inflow.

(4) If aqueous oxidant mass $>0$, compute mass balance for $N O D_{f}$ oxidation.

(5) If aqueous oxidant mass $>0$, solve second-order rate equation for $N O D_{s}$ oxidation.

(6) Update values of $F_{m t}$ and $F_{K}$.

Initial contaminant quantities in DNAPL, dissolved, and adsorbed phases may be obtained from an upscaled NAPL dissolution transport model if the ISCO model is coupled to an analytical transport model (e.g., Parker et al. 2011; Parker and Kim 2015). Otherwise, they must be estimated directly from site characterization data such as soil concentration data.

Prior to commencing ISCO, no oxidant species is assumed present. Initial quantities of total, fast and slow NOD are computed as

$m_{\text {NODtot }}=0.75 C_{\text {soil }}^{\text {NODtot }} \rho_{b} V_{T Z} / 1000$

$m_{\text {NODf }}=f_{\text {NODf }} m_{\text {NODtot }}$

$m_{N O D s}=\left(1-f_{N O D f}\right) m_{N O D t o t}$

where $m_{N O D t o t}, m_{N O D f}$, and $m_{N O D s}$ are total, fast, and slow NOD in moles $\mathrm{O}_{2}$ eq; $C_{\text {soil }}^{\text {NODtot }}$ is the total NOD quantity

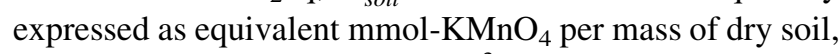
$\rho_{b}$ is the soil bulk density $\left[\mathrm{ML}^{-3}\right], V_{T Z}$ is the TZ volume $\left[\mathrm{L}^{3}\right]$, and the factor 0.75 is the ratio of mol- $\mathrm{O}_{2}$ eq to mol$\mathrm{KMnO}_{4}$.

Mass balance equations for DNAPL contaminant, aqueous and adsorbed contaminant, oxidant, chloride, and $N O D_{f}$ for each treatment zone are solved iteratively for each timestep. Advective losses of aqueous phase oxidant and contaminant are assumed to occur in proportion to the product of the average darcy velocity and current average concentration. DNAPL and NOD are assumed to be immobile. Model calculation details are given in Appendix $\mathrm{A}$ in ESM. 


\subsection{ISCO performance monitoring}

Our objective here is to formulate performance monitoring protocols to facilitate reliable real-time operational decisions to be made. To minimize decision lags due to travel times to downgradient locations, performance monitoring needs to focus on measurements within or near the aquifer volume being treated. Remediation progress is commonly monitored by measuring contaminant concentrations in soil and/or groundwater samples. But what is the relationship between these two types of measurements to each other and to the downgradient plume? To answer this question, consider a DNAPL source zone of volume $V\left[\mathrm{~L}^{3}\right]$, with an area $A_{\text {vert }}$ perpendicular to flow downgradient of the source $\left[\mathrm{L}^{2}\right]$, mean darcy velocity $q\left[\mathrm{LT}^{-1}\right]$, contaminant retardation factor $R_{C H}$, source dissolution rate versus time $J(t)$ $\left[\mathrm{MT}^{-1}\right]$, and contaminant mass remaining in the source versus time $M(t)[\mathrm{M}]$. The flow-averaged groundwater concentration $C_{a v g}^{g w}\left[\mathrm{ML}^{-3}\right]$ on the downgradient plane is

$C_{\text {avg }}^{g w}(t)=\frac{J(t)}{q R_{C H} A_{v e r t}}$

while the average soil concentration $C_{\text {avg }}^{\text {soil }}\left[\mathrm{MM}^{-1}\right]$, within the source volume is

$C_{\text {avg }}^{\text {soil }}(t)=\frac{M(t)}{\rho V}$

where $\rho$ is the soil dry density $\left[\mathrm{ML}^{-3}\right]$. Combining Eqs. (10a) and (10b) with Eq. (1) assuming no effects of oxidant $\left(F_{m t}=F_{k}=1\right)$, yields

$C_{\text {avg }}^{\text {soil }}=\frac{M_{0}}{\rho V}\left(\frac{q R_{C H} A_{\text {vert }} C_{\text {avg }}^{g w}}{J_{0}}\right)^{1 / \beta}$

which allows us to compute "equivalent" average soil or groundwater concentrations.

A complicating factor for using groundwater concentration data to monitor ISCO performance (and in using Eq. 11) is that aqueous contaminant concentrations will be negligible as long as dissolved phase oxidant is present. Following oxidant injection, aqueous phase oxidant will deplete over time due to reactions and advection and aqueous contaminant concentrations will subsequently rebound. However, full rebound can take many months, depending on the DNAPL dissolution rate and groundwater velocity. Therefore, measurements of dissolved contaminant within or near the downgradient edge of a source zone will provide limited information on the progress of remediation until rebound occurs. This will require longer waiting times between oxidant injection events to make termination and reinjection decisions than if soil sample data is used.
Since pre-existing groundwater monitoring wells will generally be present within the ISCO treatment area, which are required to be monitored at a specified interval (e.g., quarterly, semi-annual), we assume that groundwater concentration will be measured at all such wells at the regulatory-mandated intervals. Additional numbers of groundwater monitoring locations may be stipulated strictly for ISCO performance monitoring (i.e., "temporary" wells or push-probe water samples). All groundwater sampling locations will be sampled at no less than the regulatory-mandated frequency. At each groundwater sampling date following oxidant injection, oxidant concentration will be measured until oxidant concentration drops below a practical detection value $\left(C_{\text {ox }}\right.$ min $)$. Prior to reaching this level, contaminant concentrations will not be measured and afterwards, oxidant concentration will not be monitored until after the next injection event.

In addition to mandated and optional water samples, we also consider collection of soil samples for ISCO performance monitoring. Decision logic soil and groundwater data or groundwater data only are described below.

\subsection{Decision logic for ISCO termination and reinjection}

The criterion for terminating ISCO treatment is commonly specified as

$C_{\text {avg }}^{\text {type }} \leq C_{\text {stop }}^{\text {type }}$

where $C_{\text {avg }}^{\text {type }}=C_{\text {avg }}^{\text {soil }}\left[\mathrm{MM}^{-1}\right]$ or $C_{\text {avg }}^{g w}\left[\mathrm{ML}^{-3}\right]$ represents average soil or groundwater concentrations, respectively, and $C_{\text {stop }}^{\text {type }}=C_{\text {stop }}^{\text {soil }}$ or $C_{\text {stop }}^{g w}$ represents corresponding termination criterion. In practice, we never know true values of $C_{a v g}^{t y p e}$, but only estimates of the average $C_{a v g \text { smp }}^{t y p e}$ calculated from a finite number of samples. If we substitute $C_{a v g}^{\text {type }}$ smp for the true average $C_{a v g}^{t y p e}$ in Eq. (12), resulting termination decisions will have a significant probability of erroneously terminating treatment before the target criterion is actually met due to differences between $C_{\text {avg smp }}^{\text {type }}$ and $C_{\text {avg }}^{\text {type }}$.

A more conservative approach that explicitly accounts for this uncertainty is to modify the termination criteria as $C_{U C L}^{\text {type }} \leq C_{\text {stop }}^{\text {type }}$

where $C_{U C L}^{t y p e}$ is the upper confidence limit of the estimated average concentration at significance level $\alpha$ (e.g., 0.1 for a $90 \%$ upper confidence limit). Since $C_{U C L}^{t y p e}>C_{a v g s m p}^{t y p e}$ for $0<\alpha<0.5$, Eq. (13) is a more stringent criterion than Eq. (12). The ratio of $C_{U C L}^{t y p e}$ and $C_{a v g ~ s m p}^{\text {type }}$ represents a safety factor that reduces the likelihood of erroneous decisions that terminate too early. Note that $\alpha=0.5$ corresponds to $C_{U C L}^{t y p e}=C_{\text {avg smp }}^{\text {type }}$. 
High variance properties of quantities that are physically constrained to be non-negative, such as contaminant concentrations, necessarily exhibit positively skewed distributions. Normal probability distributions cannot describe such behavior. Lognormal distributions capture the major features of such data and are commonly used as a reasonable and mathematically expedient approximation. If the average concentration is estimated from $n_{s m p}^{t y p e}$ samples and a log-normal distribution is assumed, then

$C_{U C L}^{t y p e}=\exp \left(\ln \left(C_{\text {avg smp }}^{\text {type }}\right)+t_{1}(\alpha, N) S_{\ln \text { avg }}^{\text {type }}\right) \leq C_{\text {stop }}^{\text {type }}$

where

$S_{\ln a v g}^{\text {type }}=\frac{S_{\ln }^{t y p e}}{\sqrt{n_{s m p}^{t y p e}}}$

in which $C_{a v g s m p}^{\text {type }}$ is the arithmetic average of $n_{s m p}^{\text {type sam- }}$ ples, $S_{\ln }^{\text {type }}$ is the population standard deviation of $\ln$ concentration, $S_{\ln a v g}^{t y p e}$ is the standard deviation of $\ln C_{a v g s m p}^{t y p e}$, and $t_{1}(\alpha, N)$ is the one-sided $t$-value for significance level $\alpha$ with $N$ degrees of freedom (Snedecor and Cochran 1967). If $S_{\mathrm{ln}}^{t y p e}$ is computed from $n_{s m p}^{t y p e}$ samples then $N=n_{s m p}^{t y p e}-1$, while if $S_{\ln }^{t y p e}$ is based on prior site characterization data or experience with other sites, then $N=\propto$. Equation (14) may also be written

$C_{\text {avg smp }}^{\text {type }} \leq C_{\text {stop } S F}^{\text {type }}=\frac{C_{\text {stop }}^{\text {type }}}{\exp \left(t_{1}(\alpha, N) S_{\ln \text { avg }}^{\text {type }}\right)}$

where $C_{\text {stop } S F}^{\text {type }}$ is the "true" stop criterion divided by a safety factor to account for uncertainty. Note that decreasing population uncertainty $S_{\mathrm{ln}}^{\text {type }}$ and/or increasing the number of samples $n_{s m p}^{\text {type }}$ will yield lower $C_{U C L}^{t y p e}$ and higher $C_{\text {stop } S F}^{\text {type }}$ values at a given confidence level, which enable earlier ISCO termination at the specified confidence level. Alternatively, if the same termination criteria $\left(C_{U C L}^{t y p e}\right.$ and $C_{\text {stop } S F}^{\text {type }}$ ) are employed, more reliable data will result in a lower probability of erroneously terminating before actual concentrations meet the desired levels.

As an example, consider a source zone with a cleanup target of $C_{\text {stop }}^{\text {soil }}=1000 \mu \mathrm{g} / \mathrm{kg}$. It is planned to take $n_{\text {smp }}^{\text {soil }}=15$ soil samples to assess whether the objective has been met. Assume a prior estimate of $S_{\ln }^{\text {soil }}=2.9$. If we want a $95 \%$ probability $(\alpha=0.05)$ that the actual average soil concentration will be less than $1000 \mu \mathrm{g} / \mathrm{kg}$ when we terminate treatment, then $t_{1}=1.646$ and Eq. (15) indicates that the average concentration computed from 15 samples needs to be less than $C_{\text {stop } S F}^{\text {type }}=291 \mu \mathrm{g} / \mathrm{kg}$ to achieve the desired reliability. If the number of samples is increased to 30 , we could terminate earlier with the same decision confidence when $C_{\text {avg smp }}^{\text {soil }}<418 \mu \mathrm{g} / \mathrm{kg}$.

Since the time to reach $C_{\text {stop }}^{\text {type }}$ or $C_{\text {stop }}^{\text {typ }}$ will vary spatially, it may be possible to reduce operating costs by applying the foregoing criteria independently to sub-regions to terminate injection earlier in areas that reach cleanup objectives before site-wide termination criteria are met. Alternatively, we could treat less contaminated zones longer to reach a local $C_{\text {stop }}^{\text {type }}$ that is less than the site-wide value, allowing earlier termination of more contaminated areas when the site-wide criteria is met.

Anticipating that regions with higher initial contaminant concentrations are likely to take longer to cleanup, a system designer may divide a site into multiple treatment zones (TZ) based on ranges of pre-remediation contaminant concentrations observed during site characterization. When independent $\mathrm{TZ}$ termination is considered, the criteria for ISCO termination in a single TZ is taken as

$C_{\text {avg smp TZi }}^{\text {type }} \leq \frac{C_{\text {stop TZi }}^{\text {type }}}{\exp \left(t_{1}(\alpha, N) S_{\ln \text { avg } T Z i}^{\text {type }}\right)}=C_{\text {stop SF TZi }}^{\text {type }}$

$S_{\ln \text { avgTZi }}^{\text {type }}=\frac{S_{\ln T Z i}^{\text {type }}}{\sqrt{n_{\text {smp }}^{\text {soil } T Z i}}}$

where all variables are for $\mathrm{TZ} i$. Criteria for simultaneous termination of all currently operating TZs are formulated in a similar fashion, while taking into consideration that sitewide statistical properties can be described by upscaling individual $\mathrm{TZ}$ statistics as follows

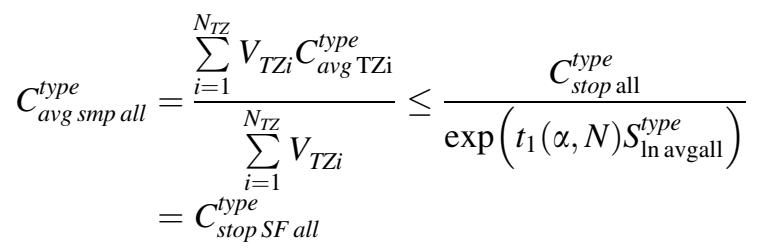

$S_{\ln \text { avg all }}^{\text {type }}=\frac{\sum_{i=1}^{N_{T Z}} n_{\text {smp } T Z i}^{\text {type }}}{\left(\sum_{i=1}^{N_{T Z}} \frac{n_{\text {spm } T Z i}^{t y z}}{\left(S_{\ln T Z i}^{\text {tpe }}\right)^{2}}\right)^{1 / 2}}$

where $V_{T Z i}$ is the bulk volume of TZ $i$. Note that the stop criterion for individual TZs is permitted to differ from that for site-wide termination, subject to the constraint that $C_{\text {stop TZi }}^{\text {type }} \leq C_{\text {stopall }}^{\text {type }}$, i.e., the TZ stop criteria cannot exceed the site-wide stop criteria. When evaluating Eq. (17) for TZs that have previously terminated operation, the average concentration and number of samples from the last sampling event prior to termination are conservatively assumed to apply. 
In addition to determining when oxidant injection can be terminated, a corollary decision must be made regarding if or when reinjection should be initiated must be made. The reinjection criteria for a given $\mathrm{TZ}$ is specified as

$C_{U C L \text { smp TZi }}^{\text {type }}>C_{\text {reinject TZi }}^{\text {type }}$

where $C_{\text {reinject } T Z i}^{\text {type }}$ is the average concentration above which reinjection is indicated $\left(C_{\text {reinject } T Z i}^{\text {type }} \geq C_{\text {stop } T Z i}^{\text {type }}\right)$, and $S_{\ln \text { avgTZi }}^{\text {type }}$ is defined by Eq. (16b). Due to the inconvenience and cost of commencing injection in TZs at different times, reinjection is not initiated until criterion for either reinjection or termination has been met for all TZs.

The time interval $\Delta t_{\text {mon }}^{g w}$ between potential groundwater sampling events for ISCO monitoring is assumed to be equal to or an integer fraction (e.g., 1, 1/2, 1/3) of the regulatory mandated interval. Following an oxidant injection event, oxidant concentration will be monitored prior to taking samples for contaminant analysis. If the oxidant concentration exceeds its detection limit for the method utilized $\left(C_{\text {ox } \min }\right)$, samples for contaminant analysis are not taken, as values would not be meaningful. Subsequently, average contaminant concentrations from groundwater samples are not deemed to represent full rebound conditions until the current average value is less than or equal to the previous value for the $\mathrm{TZ}$ or site-wide depending on the level for which the termination decision is to be made. A minimum number of groundwater sampling rounds $N_{\min }^{g w}$ is stipulated before a termination decision is allowed, where $N_{\min }^{g w}$ is at least 2. If $\Delta t_{\text {mon }}^{g w}$ is very short and/or rebound is very slow, larger $N_{\min }^{g w}$ values may be necessary to avoid erroneous early termination due to multiple rounds below detection limits prior to exhibiting rebound. The minimum number of sampling rounds is not applicable for making reinjection decisions.

To coordinate soil monitoring (when utilized) with groundwater monitoring, the period between soil sampling events $\Delta t_{\text {mon }}^{\text {soil }}$ is constrained to be an integer fraction or multiple (notated as $F_{\text {soillgw }}$ ) of $\Delta t_{\text {mon }}^{g w}$ (e.g., 1/3, 1/2, 1, 2, $3)$. Since rebound is not an issue with soil data, termination or reinjection decisions do not require a minimum number of soil sampling rounds. Operational decisions may be based on groundwater data only, soil data only, or on statistically-pooled soil and groundwater data (Appendix B in ESM) for the site as a whole or for individual TZs at the specified confidence level $\alpha$. However, to maintain sitewide coordination of injection events, reinjection in individual TZs is not implemented until all TZs have met either reinjection or termination criteria following each injection event. A flowchart of the performance monitoring and decision-making protocol given in Appendix B (Fig. B-1) in ESM.

\subsection{ISCO cost model and design optimization}

Life cycle cost to meet cleanup criteria are computed for a given set of design parameters using the model described above for a range of site characteristics (Monte Carlo realizations) generated stochastically based on uncertainty inferred from site characterization data. Since ISCO applications are typically conducted over relatively short time periods (less than a few years), an annualized discount rate (Eq. C-1a in Appendix B in ESM) was not considered in computing annual operating and monitoring costs. The ISCO cost model includes the following cost categories

$$
\begin{aligned}
\text { Total Cost }= & \text { Fixed Cost }+ \text { Operating Cost } \\
& + \text { Monitoring Cost }+ \text { Penalty Cost }
\end{aligned}
$$

where Fixed Cost is an initial charge for design and con-

struction; Operating Costs include cost per volume of fluid volume injected and per unit oxidant mass, cost per oxidant injection event, and additional cost per treatment duration (e.g., administration, reporting); Monitoring Costs include mobilization cost per sampling event, cost per monitoring well, cost per water sample, cost per soil boring, and cost per soil sample; and Penalty Cost is a cost that is incurred if the ISCO system fails to meet termination criteria within a specified timeframe. The penalty cost may be regarded as the cost to implement an alternative remediation plan if the initial design fails to perform adequately or it may be treated as merely a parameter to ensure a high probability of success (i.e., reduced risk of failure) for optimization. In either case, the penalty cost is only used for optimization calculations and is not included in reported total costs. Cost model details are given in Appendix C in ESM.

Specified design variables are optimized within defined constraints to minimize expected cost (i.e., cost averaged over 100 equal-probability Monte Carlo realizations). ISCO design variables that may be optimized include the following.

- Oxidant concentration in injected fluid per TZ, $C_{o x 0}$

- Target average groundwater or soil concentration for individual TZ termination, $C_{\text {stop }}^{\text {type }}$ TZi

- Average groundwater or soil concentration above which reinjection is initiated, $C_{\text {reinject }}^{\text {type }}$

- Number of sampling locations and depths per TZ for each data type

- Time intervals between groundwater and soil sampling events $\left(F_{\text {soil/gw }}\right), \Delta t_{m o n}^{\text {soil }} / \Delta t_{m o n}^{g w}$, and

- Minimum number of sampling rounds following injection before a termination or reinjection decision can be made, $N_{\text {min }}^{g w}$.

The ISCO model was implemented in the program SCOToolkit, which includes models for other remediation 
technologies, a 3-D dissolved transport model, and calibration modules, as well as stochastic optimization tools. When simulation is coupled with the dissolved plume model, average source zone cleanup targets for soil and/or dissolved concentration can be optimized to meet site-wide dissolved plume criteria. However, in the present study, we focus on the optimization of ISCO design to meet specified source zone cleanup criteria to identify operational and monitoring strategies that minimize total expected (i.e., probability-weighted) cost to reliably achieve cleanup objectives. Details regarding numerical implementation are given in Appendix D in ESM.

\section{Example problems}

\subsection{Example 1: ISCO performance sensitivity to aquifer parameters}

Essential ISCO design variables, such as oxidant injection rates and concentrations, are determined by considering the uncertainty of groundwater velocity and NOD parameters based on site characterization of a DNAPL site (Crimi and Siegrist 2005). Example 1 investigates the effects of those key parameters on ISCO performance for a hypothetical problem. A PCE DNAPL source is assumed within $1125 \mathrm{~m}^{2}$ area with an average thickness of $5.5 \mathrm{~m}$ corresponding to a volume of $6188 \mathrm{~m}^{3}$. Source width perpendicular to groundwater flow is $75 \mathrm{~m}$ resulting in a vertical plane area of $413 \mathrm{~m}^{2}$. Best estimates and standard deviations of DNAPL source, aquifer, and NOD parameters are summarized in Table 3 for the base case of Example 1.

It is noted that the performance of ISCO in a heterogeneous aquifer generally depends on the combined uncertainty from both site characterization and performance monitoring. To account the combined contribution of these uncertainty to decision-making in ISCO operation with regard to the success probability and cost, in Example 2 we perform Monte Carlo simulations to present the range of cost predictions while considering propagation of measurement uncertainty into each Monte Carlo simulation.

A source zone remediation target of $C_{\text {stop }}^{g w}=100 \mu \mathrm{g} / \mathrm{L}$ is assumed to yield concentrations below regulatory standards at stipulated downgradient compliance locations. This groundwater concentration corresponds to a soil concentration $\left(C_{\text {stop }}^{\text {soil }}\right)$ of $26 \mu \mathrm{g} / \mathrm{kg}$ via Eq. (11). These targets are applied to a single treatment zone. A total of 18 injection wells is used with oxidant solution injected at $16.35 \mathrm{~m}^{3} /$ day ( 3 gallons per minute) in each well until 1.25 pore volumes of oxidant solution is injected per well to ensure overlapping injection zones. Since there is only a single TZ, all injection wells are utilized during each reinjection event until the cleanup criteria is met. For all simulations in Example 1, average groundwater concentration in each TZ was determined quarterly (i.e., $\Delta t_{m o n}^{g w}=90$ days) with a minimum of two monitoring events $\left(N_{\min }^{g w}=2\right)$. Groundwater sampling commences after the oxidant concentration drops below $C_{o x} \min$ of $50 \mathrm{mg} / \mathrm{L}$ and continues until $C_{a v g}^{g w}$ exceeds $200 \mu \mathrm{g} / \mathrm{L}$, in which case oxidant reinjection is performed, or until $C_{a v g}^{g w}$ is less than or equal to $C_{\text {stop }}^{g w}$ and to $C_{a v g}^{g w}$ from the previous sampling round.

Deterministic simulations were performed to evaluate the effect of groundwater velocity and injected oxidant concentration for the following cases:

(a) $q=0.008 \mathrm{~m} /$ day and $C_{o x 0}=5 \mathrm{~g} / \mathrm{L}$,

(b) $q=0.008 \mathrm{~m} /$ day and $C_{o x 0}=25 \mathrm{~g} / \mathrm{L}$,

(c) $q=0.08 \mathrm{~m} /$ day and $C_{o x 0}=5 \mathrm{~g} / \mathrm{L}$, and

(d) $q=0.08 \mathrm{~m} /$ day and $C_{o x 0}=25 \mathrm{~g} / \mathrm{L}$.

Figure 1 shows average $\mathrm{TZ}$ concentrations versus time for each case for dissolved oxidant as green dashed lines, equilibrium dissolved PCE (asymptotic rebound computed from mass flux as cyan dashed lines, "observed" dissolved PCE (i.e., actual nonequilibrium concentrations) as solid blue lines, and soil PCE concentrations as red solid lines. All concentrations are normalized as $C_{o x}(t) / C_{o x o}$ for oxidant and as $\left(C_{\text {avg }}^{\text {type }}(t)-C_{\text {stop }}^{\text {type }}\right) /\left(C_{\text {avg }}^{\text {type }}\left(t_{0}\right)-C_{\text {stop }}^{\text {type }}\right)$ for soil
Table 3 Base case model parameters for example problems

\begin{tabular}{lll}
\hline Parameter & Best estimate & Log uncertainty $\left(S_{\text {ln }}\right)$ \\
\hline Initial source mass, $M_{o}$ & $100 \mathrm{~kg}$ & 0.2 \\
Initial dissolution rate, $J_{o}$ & $0.1 \mathrm{~kg} / \mathrm{day}$ & 0.1 \\
Depletion coefficient, $\beta$ & 0.75 & 0.1 \\
Darcy velocity, $q$ & $0.008 \mathrm{~m} / \mathrm{day}$ & 0.05 \\
Porosity, $\phi$ & 0.3 & - \\
Bulk density, $\rho_{b}$ & $1855 \mathrm{~kg} / \mathrm{m}^{3}$ & - \\
Total NOD concentration, $C_{\text {soil }}^{\text {NODto }}$ & $2.0 \mathrm{~g} / \mathrm{kg}$ & 0.3 \\
Fast NOD fraction, $f_{N O D f}$ & 0.15 & 0.3 \\
NOD rate coefficient, $k_{N O D s}$ & $0.02 \mathrm{~L} / \mathrm{mmol} \mathrm{MnO}_{4}^{-1} / \mathrm{day}$ & 0.3
\end{tabular}



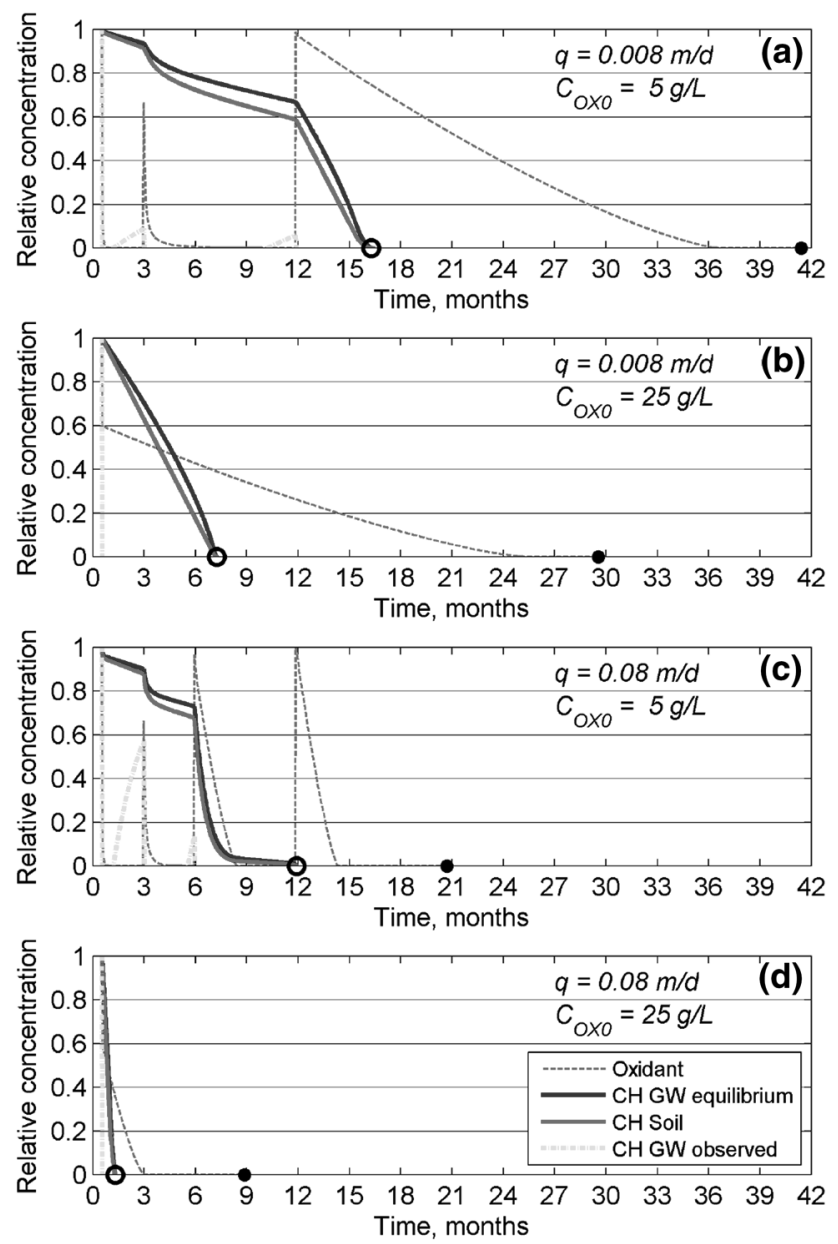

Fig. 1 Example 1 results with groundwater monitoring only and no pore clogging for a $q=0.008 \mathrm{~m} /$ day with $5 \mathrm{~g} / \mathrm{L}$ oxidant, b $q=0.008 \mathrm{~m} /$ day with $25 \mathrm{~g} / \mathrm{L}$ oxidant, c $q=0.08 \mathrm{~m} /$ day fast flow with $5 \mathrm{~g} / \mathrm{L}$ oxidant, and $\mathbf{d} q=0.08 \mathrm{~m} /$ day conditions with $25 \mathrm{~g} / \mathrm{L}$ oxidant. Empty circle $(\bigcirc)$ indicates the time when the "true" average groundwater concentration is less than $C_{\text {stop }}^{g w}=100 \mu \mathrm{g} / \mathrm{L}$ and solid circle (-) indicates the termination time based on the monitoring protocol. ' $\mathrm{CH}$ ' indicates chlorinated hydrocarbon

and groundwater contaminant concentrations, where $t_{0}$ is the time immediately prior to ISCO such that $C_{a v g}^{\text {soil }}\left(t_{0}\right)$ is $9000 \mu \mathrm{g} / \mathrm{kg}$ and $C_{a v g}^{g w}\left(t_{0}\right)$ is $7000 \mu \mathrm{g} / \mathrm{L}$ for Cases (a) and (b) and $700 \mu \mathrm{g} / \mathrm{L}$ for Cases (c) and (d).

Time series curves for Case (a) (Fig. 1a) indicate that soil and groundwater concentrations met the remediation objectives in about 16 months following three oxidant injections (open circle in Fig. 1). However, because high oxidant concentrations persisted in the treatment zone much longer due to the low velocity, the groundwater monitoring protocol was unable to confirm completion until month 41 . Three months after the first injection, the nonequilibrium groundwater contaminant concentration is predicted to rebound to only $10 \%$ of the equilibrium value. A similar percent rebound was observed in 9 months after the second injection, reflecting slower DNAPL mass transfer as DNAPL mass decreases. This phenomenon will contribute to the lag between the time remediation criteria are actually met and when it can be confirmed by groundwater monitoring.

A five-fold increase in oxidant concentration for Case (b) is predicted to reduce the time to reach the cleanup target by $50 \%$ to 7 months with only a single oxidant injection (Fig. 1b), although the groundwater monitoring protocol does not confirm termination until 30 months, due largely to slow flushing of excess oxidant from the TZ. The faster contaminant reduction is largely attributable to an increase in DNAPL mass transfer kinetics.

Case (c) with a high velocity and low oxidant concentration (Fig. 1c) exhibits more rapid rebound than Case (a), with about $85 \%$ rebound in 3 months for the first injection and $17 \%$ in 3 months for the second injection-about 8 times faster than Case (a). The faster rebound allows the third injection to be implemented sooner than for Case (a) resulting in attainment of the cleanup goal in only 12 months. However, the monitoring protocol triggered a fourth injection shortly before this, which extended the duration of monitoring to about 21 months. Due to more rapid flushing of excess oxidant at the higher velocity, the extended monitoring period was much shorter than for Cases (a) and (b).

Enhanced mass transfer rates associated with a higher oxidant concentration for Case (d) sharply accelerated remediation with actual attainment occurring in less than 2 months and confirmation from groundwater monitoring in 9 months (Fig. 1d).

The foregoing simulations were repeated with pore clogging. The mass transfer inhibition factor $\left(F_{k}\right)$ in Eq. (3), representing pore clogging effects, was computed to range from 0.96 (slow flow) to 0.97 (fast flow), indicating that mass transfer was minimally affected. Remediation duration did not increase by more than 2 days for any of the cases. These observations agree with results of Huling and Pivetz (2006) and West and Kueper (2012). It should be noted that our results are based on a value for $S_{\text {rind }}$ in Eq. (3) of $-4.6 \times 10^{-6} \mathrm{~L} / \mathrm{mg}$ reported by West et al. (2007) for a test column. However, the value of $S_{\text {rind }}$ is likely to vary for different aquifer materials, so pore clogging effects may be larger or smaller than indicated by the simulations if $S_{\text {rind }}$ exhibits substantial variability. Localized pore clogging effects have been reported in DNAPL zones and near well screens at field sites (Reitsma and Randhawa 2002). If pore clogging is a concern, it would be advisable to calibrate $S_{\text {rind }}$ from laboratory or field pilot test data.

Another geochemical factor that can affect the availability of oxidant during ISCO is the rate constant for NOD oxidation (Eqs. A-1 and A-2). Table 4 presents remediation 
times and numbers of injection events for simulations of the foregoing test problem with different groundwater velocities and NOD rate constants $\left(k_{N O D s}\right)$ for an oxidant injection concentration $\left(C_{o x 0}\right)$ of $5 \mathrm{~g} / \mathrm{L}$. The range of $k_{N O D s}$ values is based on Yan and Schwartz (2000) and Waldemer and Tratnyek (2006). NOD rate exerts a greater effect on remediation period than pore clogging especially at lower oxidant injection concentrations for the cases simulated. The NOD rate coefficient and groundwater velocity jointly affect remediation duration. At low groundwater velocities, advective oxidant loss is slow and higher NOD rates allow NOD to deplete more quickly, allowing contaminant oxidation to proceed. However, at high groundwater velocities, oxidant losses due to flushing become predominant and higher NOD rates scavenge more oxidant before it is flushed from the TZ leaving less for contaminant oxidation, increasing remediation duration.

The above simulations considered the use of groundwater sampling alone to make reinjection and termination decisions. We now reconsider Case (a) using soil and groundwater sampling, which are assumed to be taken on the same schedule. Comparison of Case (a) using groundwater data only (Fig. 1a) with that using soil and groundwater data (Fig. 2) indicates that the second oxidant injection is initiated at the same time ( 3 months after start) for both cases. However, soil monitoring triggers the third and final injection only 3 months later (6 months after start), while using the groundwater data only delayed the third injection to 12 months after start. Actual concentrations met cleanup criteria 16 months after start with groundwater data only, but only 11.5 months after start with soil data. The monitoring protocols required monitoring to continue 41 months after start using groundwater data alone but only 12 months after start with soil and groundwater data. Cost savings may or may not result depending on the savings from less groundwater sampling versus additional costs for soil sampling. However, in circumstance where remediation duration is important, soil sampling should be considered.

Table 4 Time in months to attain an aqueous PCE concentration less than $100 \mu \mathrm{g} / \mathrm{L}$ for Example 1 with various NOD rate coefficients $\left(k_{N O D s}\right)$ and groundwater velocities with $C_{o x 0}=5 \mathrm{~g} / \mathrm{L}$

\begin{tabular}{lccc}
\hline Darcy velocity & \multicolumn{4}{l}{$k_{N O D s}\left(\mathrm{~L} / \mathrm{mmol} \mathrm{MnO}_{4}^{-1} /\right.$ day $)$} \\
\cline { 2 - 4 } & 0.002 & 0.02 & 0.2 \\
\hline $0.008 \mathrm{~m} /$ day (slow) & $30.48(3)$ & $16.27(3)$ & $12.16(3)$ \\
$0.08 \mathrm{~m} /$ day (fast) & $9.93(3)$ & $11.90(4)$ & $11.93(4)$
\end{tabular}

Values in parentheses indicate the number of injection events

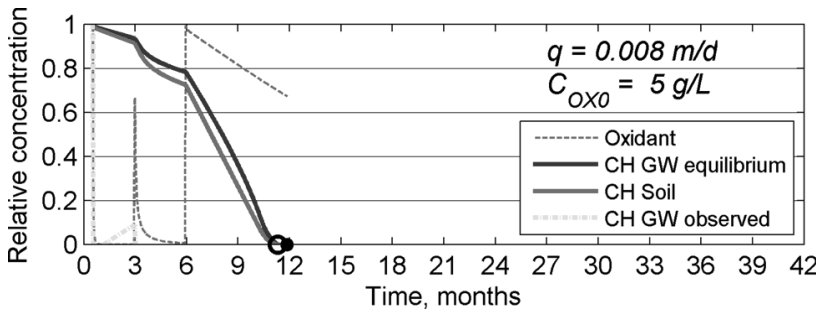

Fig. 2 Results for case shown in Fig. 1a except using soil and groundwater monitoring data. Empty circle $(O)$ indicates the time when "true" average groundwater concentration is less than $C_{\text {stop }}^{g w}=100 \mu \mathrm{g} / \mathrm{L}$ (equivalent to $C_{\text {stopall }}^{\text {soil }}=26 \mu \mathrm{g} / \mathrm{kg}$ ) and solid circle (๑) indicates the time when the system terminates based on monitoring protocol. ' $\mathrm{CH}$ ' indicates chlorinated hydrocarbon

\subsection{Example 2: Monte Carlo simulations and stochastic design optimization}

In this example, we evaluate effects of selected design variables and design approaches without and with optimization on ISCO performance and cost based on data from a PCE DNAPL-contaminated contaminated site located in North Carolina. Three TZs-A, B and C from most to least contaminated-were identified from site characterization data (Fig. 3, Table 5). Measurement uncertainty $\left(S_{l n}\right)$ was assumed to be 0.5 for individual groundwater samples and 1.15 for soil samples when taken to simulate "noisy" soil and groundwater performance monitoring data. For all cases, groundwater samples were assumed to be taken quarterly $\left(\Delta t_{\text {mon }}^{g w}=90\right.$ days) from existing compliance monitoring wells. Four monitoring location are assumed in each TZ. Following each oxidant injection, oxidant concentration was determined on the quarterly schedule. Contaminant samples were not collected until the oxidant concentration dropped below $C_{\text {ox } \min }$ taken to be $50 \mathrm{mg} / \mathrm{L}$ for all cases. Soil sampling and additional groundwater sampling were considered for selected cases.

The objective for this example problem is to compare expected cost-to-complete and failure probability for various unoptimized (conventional) designs and for designs

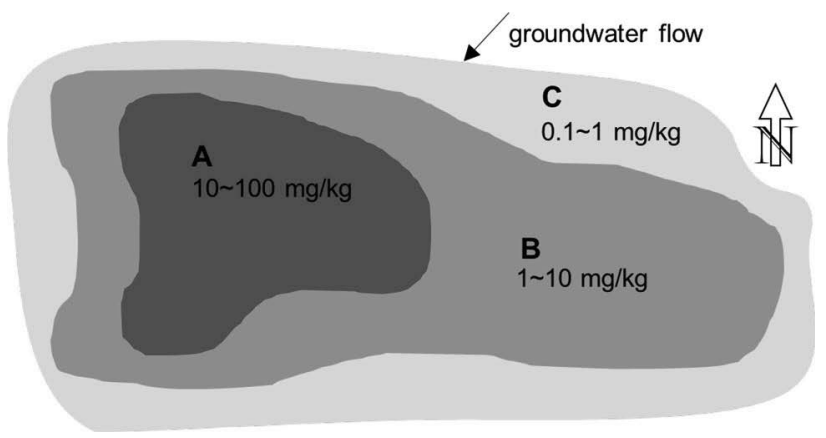

Fig. 3 Configuration of treatment zones for Example 2 
Table 5 Treatment zones and initial PCE concentrations for Example 2

\begin{tabular}{lllll}
\hline TZ & Area $\left(\mathrm{m}^{2}\right)$ & Width $(\mathrm{m})$ & Thickness $(\mathrm{m})$ & Soil concentration $(\mathrm{mg} / \mathrm{kg})$ \\
\hline A & 200 & 25 & 5.5 & $10-100$ \\
B & 460 & 60 & 5.5 & $1-10$ \\
C & 465 & 75 & 5.5 & $0.1-1$ \\
All & 1125 & 75 & 5.5 & $0.1-100$ \\
\hline
\end{tabular}

determined using stochastic optimization. SCOToolkit was used to perform stochastic optimization and to assess performance uncertainty for both optimized and nonoptimized designs. Stochastic optimization identifies design parameters that minimize expected (i.e., probability-weighted average) total remediation cost to meet specified remediation criteria considering uncertainty in model predictions and monitoring data. Uncertainty in remediation performance and cost are quantified by Monte Carlo simulations for a given set of design variables (Appendix $\mathrm{C}$ and Fig. B-1, respectively in ESM). One hundred Monte Carlo realizations of model parameters were generated assuming log-normal distributions of parameters in Table 3, in line with previous studies (Cardiff et al. 2010; Parker et al. 2010b; Kim et al. 2013; Parker et al. 2017). SCOToolkit is capable of coupling effects of source mass reduction technologies (e.g., ISCO, thermal source reduction) to downgradient dissolved plume attenuation. However, our focus here is on optimization of monitoring parameters to meet specified source cleanup objectives without direct consideration of downstream plume behavior. A total of 8 cases was investigated with four unoptimized Monte Carlo simulations (NoOpt1-NoOpt4) and four stochastic optimization cases (Opt1-Opt4), all using the same parameter set realizations. Assumed unit costs used for all simulations are summarized in Table 6. Details of the cost model are described in Appendix C in ESM.

Table 6 Unit costs for Example 2

\begin{tabular}{llllll}
\hline Parameter & Unit cost & & Parameter & \multicolumn{2}{l}{ Unit cost } \\
\hline$\$_{\text {base }}$ & 108.16 & $\$ \mathrm{k}$ & $\$_{\text {smp } 0}^{\text {GPsoil }}$ & 0.90 & $\$ \mathrm{k} /$ sample \\
$\$_{\text {mass }}$ & 0.0055 & $\$ \mathrm{k} / \mathrm{kg}$ & $\$_{\text {smp } 1}^{G P \text { soil }}$ & 0.30 & $\$ \mathrm{k} /$ sample \\
$\$_{\text {vol }}$ & 0.02076 & $\$ \mathrm{k} / \mathrm{m}^{3}$ & $\$_{\text {OXsmp }}$ & 0.10 & $\$ \mathrm{k} / \mathrm{event} / \mathrm{TZ}$ \\
$\$_{\text {time }}$ & 0.30 & $\$ \mathrm{k} / \mathrm{day}$ & $\$_{\text {penalty }}$ & $10^{6}$ & $\$ \mathrm{k} /$ failure \\
\hline
\end{tabular}

$\$$ base : fixed cost excluding other itemized cost variables, $\$$ mass: cost per unit oxidant mass injected, $\$_{\mathrm{vol}}$ : cost per volume of injected fluid excluding \$mass, $\$$ time: cost per unit time for project management, reporting, etc., $\$_{\text {smp } 0}^{G P_{s o i l}}$ : cost to collect and analyze of first soil sample depth, $\$_{s m p 1}^{G P_{\text {soil }}}$ : cost to collect and analyze of each additional sample depth at same time, $\$_{\text {OXsmp }}$ : cost per oxidant measurement, $\$_{\text {penalty }}$ : cost incurred if the system fails to meet termination criteria within a specified timeframe
Fixed and optimized design variables for all cases are summarized in Table 7. Design variables for NoOpt1 were selected to be representative of current "best engineering practice." Other NoOpt cases consider sensitivity of performance to specific design variables. All unoptimized cases terminate ISCO treatment independently for each TZ with a fixed termination criterion $C_{\text {stop } T Z}^{g w}$ of $100 \mu \mathrm{g} / \mathrm{L}$. A minimum of two sampling rounds after each injection is stipulated before a termination or reinjection decision can be made. The termination criteria for NoOpt 1 compares the average measured groundwater concentration in each $\mathrm{TZ}$ to the cleanup target without consideration of uncertainty in the average. NoOpt2, NoOpt3, and NoOpt 4 employ more stringent termination criteria with NoOpt3 and NoOpt4 using 95\% upper confidence limits of average concentrations for termination, and NoOpt 2 and NoOpt 4 requiring more groundwater sampling events (Table 7).

Optimized cases are designed to evaluate the performance of remediation systems using stochastic optimization to determine various design variables. All four cases, Opt1-Opt4, optimize the number of groundwater monitoring wells $\left(N_{M \text { Wnew }}\right)$, groundwater concentration targets for termination and reinjection decisions $\left(C_{\text {stop }}^{g w}\right.$ and $C_{\text {reinject }}^{g w}$, and injected oxidant concentrations for each $\mathrm{TZ}$ $\left(C_{\mathrm{ox} 0} \mathrm{TZ}\right)$. Opt1 and Opt2 assume groundwater monitoring only, while Opt 3 and Opt 4 optimize the number of soils borings per TZ $\left(N_{\text {loc } / T Z}\right)$ and the number of soil samples per boring $\left(N_{\text {smp/loc }}\right)$. Opt1 and Opt3 use mean values of monitoring data to make decisions $(\alpha=0.5)$, while Opt2 and Opt 4 use 95\% upper confidence limits $(\alpha=0.05)$. All optimized cases assume a minimum of 2 sampling events prior to making termination or reinjection decisions. Optimized designs terminate ISCO injection for all TZs when the site-wide groundwater concentration confidence limit is below $C_{\text {stop all }}^{g w}=100 \mu \mathrm{g} / \mathrm{L}$, and terminate individual TZs when the TZ groundwater concentration confidence limit is below $C_{\text {stop } T Z}^{g w}$, which is optimized subject to the constraint that it cannot exceed $C_{\text {stop TZ }}^{g w}$. For cases with soil sampling, termination may occur at a soil concentration confidence limit corresponding $C_{\text {stop }}^{\text {soil }}$ value computed from Eq. (11). The $C_{\text {stop }}^{\text {soil }}$ value corresponding to best estimates of model parameters is $26 \mu \mathrm{g} / \mathrm{kg}$. Note, however, that $C_{\text {stop }}^{\text {soil }}$ will vary for each Monte Carlo realization depending on 
Table 7 Results for Example 2 unoptimized design (NoOpt1-NoOpt4) and stochastic optimization scenarios (Opt1-Opt4)

\begin{tabular}{|c|c|c|c|c|c|c|c|c|c|}
\hline \multicolumn{2}{|l|}{ Case } & NoOpt1 & NoOpt2 & NoOpt3 & NoOpt4 & Opt1 & Opt2 & Opt3 & Opt4 \\
\hline \multicolumn{2}{|l|}{$\overline{\text { ENPV (\$k) }}$} & 1034 & 1062 & 1035 & 1066 & 955 & 959 & 952 & 957 \\
\hline \multicolumn{2}{|l|}{ Adjusted ENPV (\$k) ${ }^{1}$} & 1077 & 1073 & 1067 & 1066 & 955 & 959 & 952 & 957 \\
\hline \multicolumn{2}{|l|}{ Failure probability (\%) } & 4 & 1 & 3 & $<1$ & $<1$ & $<1$ & $<1$ & $<1$ \\
\hline \multicolumn{2}{|l|}{ Expected $C_{g w}(\mu \mathrm{g} / \mathrm{L})$} & 50 & 2 & 49 & 1 & 6 & 5 & 11 & 4 \\
\hline \multicolumn{2}{|l|}{ Expected duration (yrs) } & 4.20 & 4.29 & 4.16 & 4.29 & 3.93 & 3.89 & 4.07 & 4.20 \\
\hline \multicolumn{2}{|l|}{ Expected oxidant vol $\left(\mathrm{m}^{3}\right)$} & 5260 & 5344 & 5335 & 5410 & 4293 & 4680 & 3952 & 3845 \\
\hline \multicolumn{2}{|l|}{ Expected oxidant mass $(\mathrm{kg})$} & 52.6 & 53.4 & 53.3 & 54.1 & 46.2 & 46.1 & 47.6 & 48.0 \\
\hline \multirow{3}{*}{$\begin{array}{l}\text { Expected number of } \\
\text { injection events }\end{array}$} & $\mathrm{TZ}_{\mathrm{A}}$ & 4.7 & 4.8 & 4.8 & 4.9 & 4.5 & 4.6 & 3.7 & 3.5 \\
\hline & $\mathrm{TZ}_{\mathrm{B}}$ & 1.9 & 1.9 & 1.9 & 1.9 & 1.4 & 1.6 & 1.3 & 1.4 \\
\hline & $\mathrm{TZ}_{\mathrm{C}}$ & 1.6 & 1.6 & 1.7 & 1.7 & 1.2 & 1.3 & 1.3 & 1.1 \\
\hline \multirow{3}{*}{$\begin{array}{l}\text { Number of monitoring } \\
\text { wells per TZ }\end{array}$} & $\mathrm{T} Z_{\mathrm{A}}$ & 4 & 4 & 4 & 4 & $\underline{4}$ & $\underline{4}$ & $\underline{4}$ & $\underline{4}$ \\
\hline & $\mathrm{TZ}_{\mathrm{B}}$ & 4 & 4 & 4 & 4 & $\underline{4}$ & $\underline{4}$ & $\underline{4}$ & $\underline{4}$ \\
\hline & $\mathrm{TZ}_{\mathrm{C}}$ & 4 & 4 & 4 & 4 & $\underline{4}$ & $\underline{4}$ & $\underline{4}$ & $\underline{4}$ \\
\hline \multirow{3}{*}{$\begin{array}{l}\text { Number of soil borings } \\
\text { per TZ }\end{array}$} & $\mathrm{TZ}_{\mathrm{A}}$ & $\mathbf{0}$ & $\mathbf{0}$ & $\mathbf{0}$ & $\mathbf{0}$ & $\mathbf{0}$ & $\mathbf{0}$ & $\underline{0}$ & $\underline{0}$ \\
\hline & $\mathrm{TZ}_{\mathrm{B}}$ & $\mathbf{0}$ & $\mathbf{0}$ & $\mathbf{0}$ & $\mathbf{0}$ & $\mathbf{0}$ & $\mathbf{0}$ & $\underline{1}$ & $\underline{1}$ \\
\hline & $\mathrm{TZ}_{\mathrm{C}}$ & $\mathbf{0}$ & $\mathbf{0}$ & $\mathbf{0}$ & $\mathbf{0}$ & $\mathbf{0}$ & $\mathbf{0}$ & $\underline{2}$ & $\underline{1}$ \\
\hline \multicolumn{2}{|l|}{ Soil samples per boring } & $\mathbf{0}$ & $\mathbf{0}$ & $\mathbf{0}$ & $\mathbf{0}$ & $\mathbf{0}$ & $\mathbf{0}$ & $\underline{1}$ & $\underline{2}$ \\
\hline \multicolumn{2}{|l|}{ Min. sampling events } & 2 & 3 & 2 & 3 & 2 & 2 & 2 & 2 \\
\hline \multicolumn{2}{|c|}{ Soil sampling frequency $\left(F_{\text {soil/gw }}\right)$} & $\mathbf{0}$ & $\mathbf{0}$ & $\mathbf{0}$ & $\mathbf{0}$ & $\mathbf{0}$ & $\mathbf{0}$ & $\underline{3}$ & $\underline{3}$ \\
\hline \multicolumn{2}{|l|}{ Significance level, $\alpha$} & 0.50 & 0.50 & 0.05 & 0.05 & 0.50 & 0.05 & 0.50 & 0.05 \\
\hline \multicolumn{2}{|l|}{$C_{\text {stop } T Z}^{g w}(\mu \mathrm{g} / \mathrm{L})$} & 100 & 100 & 100 & 100 & $\underline{95}$ & $\underline{25}$ & $\underline{47}$ & $\underline{94}$ \\
\hline \multicolumn{2}{|l|}{$C_{\text {reinject }}^{g w}(\mu \mathrm{g} / \mathrm{L})$} & 200 & 200 & 200 & 200 & $\underline{306}$ & $\underline{269}$ & $\underline{344}$ & $\underline{339}$ \\
\hline \multirow{3}{*}{$\mathrm{C}_{o x 0}(\mathrm{~g} / \mathrm{L})$} & $\mathrm{T} Z_{\mathrm{A}}$ & 10.0 & 10.0 & 10.0 & 10.0 & 7.0 & $\underline{6.4}$ & 9.2 & $\underline{8.4}$ \\
\hline & $\mathrm{TZ}_{\mathrm{B}}$ & 10.0 & 10.0 & 10.0 & 10.0 & $\underline{13.2}$ & $\underline{11.6}$ & $\underline{15.5}$ & $\underline{14.5}$ \\
\hline & $\mathrm{TZ}_{\mathrm{C}}$ & 10.0 & 10.0 & 10.0 & 10.0 & $\underline{14.1}$ & $\underline{13.0}$ & $\underline{12.2}$ & $\underline{15.5}$ \\
\hline
\end{tabular}

${ }^{1}$ Adjusted ENPV = no penalty ENPV / (1-Failure probability/100).

Bold $=$ fixed variables, $\underline{\text { underlined }}=$ optimized variables, others $=$ computed results

stochastic values for source parameters and groundwater velocity (Eq. 11). The oxidant reinjection criterion $C_{\text {reinject }}^{g w}$ was taken as $200 \mu \mathrm{g} / \mathrm{L}$ for unoptimized cases and was optimized for other cases. The concentrations of injected oxidant $C_{o x 0}$ were assumed to be $10 \mathrm{~g} / \mathrm{L}$ for all TZs for unoptimized cases, but were optimized for the optimized cases.

In Table 7, results of all Example 2 cases include the expected net present value (ENPV) cost (Eq. C-1 in Appendix C in ESM), probability of average groundwater concentration failing to meet the target value, failure-adjusted ENPV cost, expected average groundwater concentration, expected treatment duration, expected total volume of oxidant solution, and expected total mass of injected $\mathrm{KMnO}_{4}$. ENPV costs in Table 7 are probabilityweighted total costs averaged across Monte Carlo simulations excluding penalty costs. Also tabulated is an adjusted ENPV cost, which is the ENPV cost divided by the probability of successful completion (= 1-failure probability). The latter is a normalized measure of cost to compare design alternatives with the probability of failure (i.e., risk) taken into account. Probability distributions of NPV cost excluding penalty costs are illustrated in Fig. 4 for all NoOpt and Opt cases. 

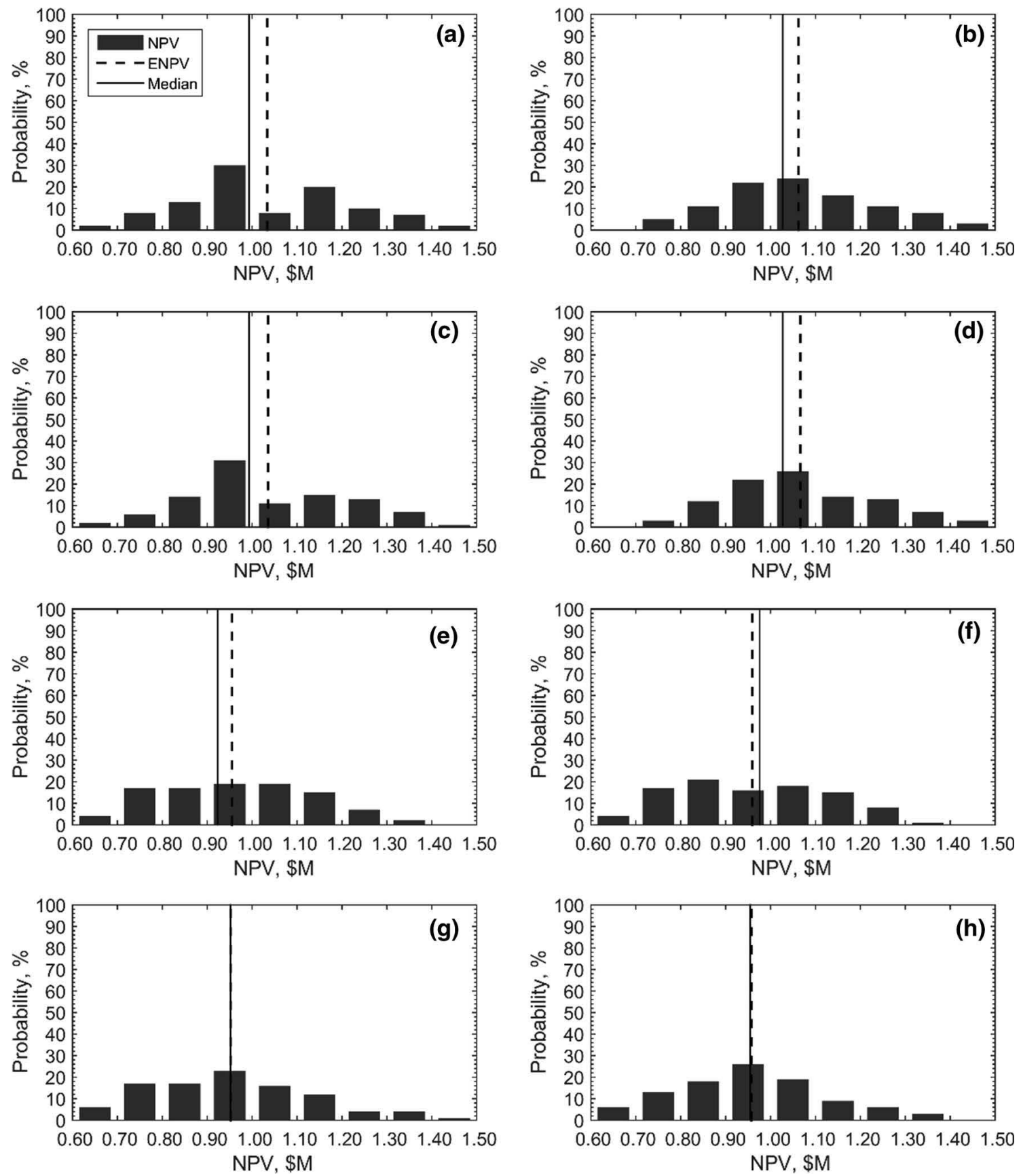

Fig. 4 Probability distributions of unadjusted NPV total cost excluding penalty cost for Example 2 cases: a NoOpt1, b NoOpt2, c NoOpt3, d NoOpt4, e Opt1, f Opt2, g Opt3, and h Opt4

NoOptl Results NoOpt1 had a $4 \%$ failure probability (i.e., probability of $C_{\text {avg all }}^{\text {gw }}>100 \mu \mathrm{g} / \mathrm{L}$ ) with an ENPV cost of $\$ 1,034 \mathrm{k}$ and an adjusted ENPV cost of $\$ 1,077 \mathrm{k}$. Inspection of the 4 NoOpt 1 Monte Carlo realizations that failed to meet actual remediation criteria (based on "true" noise-free simulations) indicated that three of the four failures occurred because observed aqueous concentrations in one $\mathrm{TZ}$ remained at non-detect for the required minimium of two quarterly sampling events, thus triggering
ISCO termination for that TZ. However, the actual full rebound concentration was much above the cleanup target. The erroneous early terminations were thus attributable to slow rebound.

NoOpt2 Results This case is identical to NoOpt1, except that 'the minimum number of sampling events' following each injection event was increased from 2 to 3 to avoid erroneous early termination decisions noted in NoOpt1. Increasing the minimum number of sampling rounds 
eliminated the three erroneous early termination decisions associated with slow rebound in the NoOpt 1 case, leaving only 1 noncompliant realization ( $1 \%$ failure probability). The reduced failure probability comes at the expense of an increase in the ENPV cost to $\$ 1,062 \mathrm{k}$ with a slightly lower adjusted ENPV cost of $\$ 1,073 \mathrm{k}$.

NoOpt 3 and NoOpt4 Results These two cases are identical to NoOpt1 and NoOpt2, respectively, except that a significance level $\alpha$ of 0.05 was used rather than 0.5 -i.e., the $95 \%$ UCL of average measured concentration rather than the average itself was compared with the target level to make termination decisions. NoOpt 3 with a minimum of 2 sampling rounds has a $3 \%$ failure, which corresponds to the three Monte Carlo realizations that failed in NoOpt 1 due to slow rebound. Using the lower $\alpha$ value of 0.05 in NoOpt 3 eliminated the single NoOpt2 failure case, which was attributable to average measured concentrations substantially less than actual averages because of measurement "noise." NoOpt4, with a minimum of three sampling rounds, has a failure probability $<1 \%$ (i.e., less than the resolution of 100 Monte Carlo realizations). Using a higher minimum number of sampling events for NoOpt 4 eliminated the remaining failure realizations associated with slow rebound. The ENPV and adjusted ENPV costs for NoOpt 4 were $\$ 1,066 \mathrm{k}$.

Opt1 and Opt2 Results These simulations optimize the number of groundwater monitoring wells from a minimum of 4 in each TZ (currently available compliance wells) to a maximum of 10 in each TZ. Quarterly sampling is assumed. No soil sampling is considered. A fixed value of two is specified for the minimum number of groundwater sampling rounds following each injection before termination or reinjection decisions can be made. Fixed values of $\alpha=0.5$ for Opt 1 and $\alpha=0.05$ for Opt 2 are assumed. Additional optimized variables for these cases are injected oxidant concentrations for each TZ, average groundwater concentration below which ISCO can be terminated for an individual $\mathrm{TZ} C_{\text {stop } T Z}^{g w}$, and average groundwater concentration above which oxidant reinjection will be intitated for a TZ $C_{\text {reinject }}^{g w}$. Note that since $\alpha$ and $C_{\text {stop } T Z}^{g w}$ are explicitly related via Eq. (14), both cannot be optimized.

Results for Opt 1 indicate a failure probability of $<1 \%$ (Table 7). The ENPV cost is $\$ 955 \mathrm{k}$, which is $\$ 79 \mathrm{k}$ (7.6\%) lower than that for NoOpt1, the "best engineering practice" case, which had a $4 \%$ failure probability. The adjusted NPV cost for Opt1 is $\$ 122 \mathrm{k}(11.3 \%)$ lower than that for NoOpt1. Compared to NoOpt4, which had a failure probability $<1 \%$, the Opt 1 ENPV cost is $\$ 111 \mathrm{k}(10.4 \%)$ lower. Savings for Opt 1 are achieved by a 3 month shorter expected duration, $18 \%$ lower total oxidant volume, and $12 \%$ lower oxidant mass utilized compared to NoOpt 1 .
Optimization of the number of monitoring wells for Opt1 kept the number at their initial values of four per TZ. The optimized oxidant concentration was $7 \mathrm{~g} / \mathrm{L}$ for TZ A (the smallest, most contaminanted zone) and about $14 \mathrm{~g} / \mathrm{L}$ for TZs B and C. The TZ stop criteria $C_{\text {stop } T Z}^{g w}$ was slightly more aggressive $(95 \mu \mathrm{g} / \mathrm{L})$ compared to the site-wide value $C_{\text {stopall }}^{g w}(100 \mu \mathrm{g} / \mathrm{L})$ and the optimized $C_{\text {reinject }}^{g w}$ value of $306 \mu \mathrm{g} / \mathrm{L}$ was significantly more aggressive than the value used for the NoOpt cases $(200 \mu \mathrm{g} / \mathrm{L})$. The probabilityweighted average number of oxidant injection events was 4.5 for $\mathrm{TZ} \mathrm{A}, 1.4$ for $\mathrm{TZ} \mathrm{B}$ and 1.2 for $\mathrm{TZ} \mathrm{C}$.

Results for Opt 2 with $\alpha=0.05$ differ little from Opt 1 . The failure probabilities were both $<1 \%$ and the ENPV cost of Opt 2 was only $\$ 4 \mathrm{k}$ higher than Opt 1 . The more stringent $\alpha$ value used for Opt 2 was offset by slightly less aggressive optimized TZ oxidant concentrations and a less aggressive $C_{\text {reinject }}^{g w}$, while the optimized value of $C_{\text {stop TZ }}^{g w}$ was more aggressive than for Opt1. Interactions among the optimized variables are clearly complex and nonlinear.

Opt 3 and Opt4 Results These cases are the same as Opt1 and Opt2, except that soil sampling is considered in addition to groundwater monitoring. We still assume existing groundwater monitoring wells will be sampled and allow additional wells (up to 10 per TZ) to be installed. From 0 to 10 soil borings are also allowed for each $\mathrm{TZ}$ with up to 2 sample depths per boring. The frequency of soil sampling as a multiple of the quarterly frequency of groundwater sampling was also optimized between once a quarter to once every 4 quarters.

Opt3 with $\alpha=0.5$ yielded a failure probability $<1 \%$ and an ENPV cost of $\$ 952 \mathrm{k}$, just $\$ 3 \mathrm{k}$ less than Opt1. Opt4 with $\alpha=0.05$ also had a failure probability $<1 \%$. Its ENPV cost was $\$ 957 \mathrm{k}$, slightly higher than Opt1 and slightly lower than Opt2. The optimized number of monitoring wells for both Opt3 and Opt 4 was 4 for each TZ, corresponding to the initial wells available. The optimized number of soil borings was zero in TZ A and one in TZ B for both cases. The optimized number of soil borings for $\mathrm{TZ} \mathrm{C}$ was two for Opt3 and one for Opt4. In addtion, the optimized frequency of soil sampling $\left(F_{\text {soillgw }}\right)$ was once every three groundwater samplings for both Opt3 and Opt4, i.e., optimized $F_{\text {soillgw }}=3$ indicating $\Delta t_{\text {mon }}^{\text {soil }}=3$ $\Delta t_{\text {mon }}^{g w}$.

Optimized oxidant concentrations for each $\mathrm{TZ}$ were higher for Opt 3 and Opt 4 than for Opt 1 and Opt2, resulting in fewer oxidant injections for Opt 3 and Opt 4 compared to Opt1 and Opt2. However, the average treatment duration for Opt3 and Op4 were longer due to longer intervals between injection events to perform additional sampling. As observed for Opt1 and Opt2, similar cost and performance was achieved for Opt 3 and Opt 4 by optimizing $C_{\text {stop TZ }}^{g w}, \quad C_{\text {reinject }}^{g w}, C_{\text {oxo } 0}$, and performance monitoring 
Table 8 Probability of exceeding failure-adjusted costs for NoOpt1 and Opt4 cases

\begin{tabular}{lcc}
\hline Adjusted cost $(\$ \mathrm{k})$ & NoOpt1 $(\%)$ & Opt4 $(\%)$ \\
\hline 600 & 100 & 100 \\
700 & 98 & 94 \\
800 & 95 & 81 \\
900 & 82 & 63 \\
1000 & 61 & 37 \\
1100 & 41 & 17 \\
1200 & 27 & 9 \\
1300 & 16 & 3 \\
1400 & 5 & 0 \\
1500 & 1 & 0 \\
1600 & 0 & 0 \\
\hline
\end{tabular}

variables, regardless of the assumed fixed value of $\alpha$. We regard Opt 4 as the best case for optimization as it permits soil sampling to the extent justified by performance and cost and uses a conservative significance level which reduces failure probability.

Inspection of cost probability distributions for the various cases (Fig. 4) reveals a distinct positive skew for all NoOpt cases as evidenced by expected values that are significantly greater than the medians. This is much less the case for optimized simulations, which exhibit essentially zero skew for Opt3 and Opt4, slightly negative skew for Opt2, and positive skew for Opt1 (which was largely constrained by NoOpt assumptions). Narrowing our attention to the "current best practice" case (NoOpt1) and the most conservative optimization case (Opt4), we consider the probability of exceeding various adjusted total costs for these cases (Table 8). Minimum costs are nearly identical for both cases. However, the adjusted expected cost for Opt4 is $\$ 120 \mathrm{k}$ less than that for NoOpt1, the maximum adjusted cost is $\$ 156 \mathrm{k}$ less. Thus, optimization not only reduced the adjusted expected cost by $11.1 \%$, but reduced the worst-case cost by an even greater amount (14.5\%).

\section{Summary and conclusions}

We have presented a simplified model for DNAPL source remediation using ISCO that incorporates the most important physical and chemical processes with a variety of performance monitoring options for making real-time decisions and implementing most cost-effective solutions. Stochastic cost optimization is employed to determine design variables that minimize the expected total cost to achieve defined remediation objectives. Findings can be summarized as follows.

(1) Higher groundwater velocities, NOD reaction rate coefficients, and injected oxidant concentrations decreased the duration of ISCO to achieve cleanup objectives for a hypothetical case study.

(2) Pore clogging was not found to have a significant effect for the conditions studied using a literature value for the pore clogging coefficient $S_{\text {rind }}$. However, as little information is available on the variability of this coefficient, we suggest field data be collected to calibrate the value if pore clogging is a concern.

(3) Cases studied that had $<1 \%$ probability of failing to meet cleanup criteria showed post-remediation average groundwater concentrations ranging from 1 to $11 \mu \mathrm{g} / \mathrm{L}$ (see expected $\mathrm{C}_{\mathrm{gw}}$ in Table 7)-far below the cleanup target of $100 \mu \mathrm{g} / \mathrm{L}$. This implies that if one rigorously designed for average site conditions, the probability of failure would be very high. Simulation results reported here clearly demonstrate the importance of accounting for uncertainty in site characterization and monitoring data in the design process.

(4) A unique feature of the proposed ISCO operational methodology is the introduction of a termination criteria that compares the upper confidence limit of average measured concentration at a specified probability level with the cleanup target to provide a margin of safety to termination decisions. Derived statistical termination criteria allow site-wide and treatment zone termination decisions to be made with equal reliability. In some cases, cleaning up less contaminated TZs to more stringent criteria can allow site-wide average concentration targets to be met earlier and with lower costs.

(5) Non-optimized cases revealed that "noisy" measurements and limited numbers of samples can lead to termination decisions before actual cleanup criteria are met. The likelihood of such errors can be reduced by using upper confidence limits of average measured concentrations at a suitable significance level and/or using TZ stop criteria for soil or groundwater that is less than the site-wide criteria, i.e., $C_{\text {stop } T Z}^{g w}<$ $C_{\text {stop all }}^{g w}$. Considering the complexity of optimized parameter interactions evident in the optimization examples and the fact that confidence level probability and $C_{\text {stop TZ }}^{g w}$ (or $C_{\text {stop TZ }}^{\text {soil }}$ ) cannot be simultaneously optimized, we suggest to use a modest fixed value for $\alpha$ (e.g., 0.2 corresponding to an $80 \%$ upper confidence limit) and optimize $C_{\text {stop } T Z}^{g w}$ values. 
(6) If a minimum of groundwater sampling events $\left(N_{\min }^{g w}\right)$ after oxidant injection is required before making termination decisions, but it takes more than $N_{\text {min }}^{g w}$ rounds before the concentration rebounds above the termination criteria, the decision will be in error.

(7) Stochastic optimization yielded a failure-adjusted expected cost about $11 \%$ lower than a non-optimized case representative of current best engineering practice. Furthermore, optimization reduced positive skew evident in the "best practice" case such that the worst case cost for the optimized design was $14.5 \%$ lower than that for the non-optimized design (Fig. 4). Since the number of monitoring wells assumed for the "best practice" case was greater than is often available, and the assumed number fortuitously turned out to be optimal, significantly larger cost savings are likely in many cases.

Because cost and performance sensitivity are highly dependent on many site-specific factors, we do not want to leave the impression that optimal ISCO operating or performance monitoring variables can be inferred from the specific cases presented here. However, adoption of the stochastic design process in conjunction with proposed real-time performance monitoring and decision-making protocols promises to yield more robust, reliable and costeffective applications of ISCO to DNAPL sites.

Costs for ISCO have been reported to range from $\$ 26$ to $\$ 679$ per $\mathrm{m}^{3}$ with median values between $\$ 123$ and $\$ 163$ per $\mathrm{m}^{3}$ (McDade et al. 2005; Krembs et al. 2010). The extremely wide range in reported volumetric costs likely reflects large variations in contaminant properties, initial concentrations, target cleanup levels, source depth and thickness, geologic complexity, etc. For the Monte Carlo simulations reported here, average costs ranged from about $\$ 115$ to $\$ 225$ per $\mathrm{m}^{3}$, which is well within the reported range.

The ISCO model discussed here is part of a comprehensive tool for multi-strategy DNAPL site remediation design, which includes additional modules for thermal treatment and enhanced bioremediation coupled with a semi-analytical 3-D dissolved phase plume model for multiple DNAPL sources, calibration tools, and a stochastic cost optimization module to assess the performance of multiple concurrent and/or serial remediation technologies. The program, referred as the Stochastic Cost Optimization Toolkit (SCOToolkit), is publicly available at http://scotoolkit.csuohio.edu/index.php.

\footnotetext{
Acknowledgements This research was conducted with funding from the U.S. Department of Defense Strategic Environmental Research and Development Program (SERDP) Environmental Restoration Program managed by Dr. Andrea Leeson under project ER-2310 entitled "A practical approach for remediation performance
}

assessment and optimization at DNAPL sites for early identification and correction of problems."

\section{References}

ASTM (2007) Standard test method for estimating the permanganate natural oxidant demand of soil and aquifer solids, method D7262-07. ASTM International, West Conshohocken

Bastante FG, Taboada J, Alejano L, Alonso E (2008) Optimization tools and simulation methods for designing and evaluating a mining operation. Stoch Environ Res Risk Assess 22:727-735. https://doi.org/10.1007/s00477-007-0182-6

Borden R, Cha KY, Simpkin T, Lieberman MT (2010) Development of a design tool for planning aqueous amendment injection systems permanganate design tool. Final report. ESTCP Project ER-200626

Cardiff M, Liu X, Kitanidis PK, Parker J, Kim U (2010) Cost optimization of DNAPL source and plume remediation under uncertainty using a semi-analytic model. J Contam Hydrol 113(1-4):25-43. https://doi.org/10.1016/j.jconhyd.2009.11.004

Cha KY (2012) Development of design tools for in situ remediation technologies. PhD dissertation, Civil Engineering, North Carolina State University

Cha KY, Borden RC (2012) Impact of injection system design on ISCO performance with permanganate-mathematical modeling results. J Contam Hydrol 128:33-46

Crimi ML, Siegrist RL (2005) Factors affecting effectiveness and efficiency of DNAPL destruction using potassium permanganate and catalyzed hydrogen peroxide. J Environ Eng 131(12):1724-1732

EPA (2000) Subsurface remediation: improving long-term monitoring \& remedial systems performance. EPA/542/B-00/002. In: Conference proceedings. June 8-11, 1999. US Environmental Protection Agency, St. Louis, MI, $81 \mathrm{pp}$

EPA (2007) Long-term groundwater monitoring optimization newark, muscoy, and source operable units newmark superfund sites San Bernardino, California. EPA 542-R-07-015. Report. US Environmental Protection Agency, $326 \mathrm{pp}$

Falta RW, Rao PS, Basu N (2005) Assessing the impacts of partial mass depletion in DNAPL source zones: I. Analytical modeling of source strength functions and plume response. J Contam Hydrol 78:259-280

Freeze A (2004) The role of stochastic hydrogeological modeling in real-world engineering applications. Stoch Environ Res Risk Assess 18:286-289. https://doi.org/10.1007/s00477-004-0194-4

Huling SG, Pivetz BE (2006) In situ chemical oxidation. EPA engineering issue (EPA/600/R-06/072)

Jawitz JW, Fure AD, Demmy GG, Berglund S, Rao PSC (2005) Groundwater contaminant flux reduction resulting from nonaqueous phase liquid mass reduction. Water Resour Res 41(10):W10408

Kim U, Parker J, Kitanidis P, Cardiff M, Liu X, Gillie J (2013) Stochastic cost optimization of DNAPL remediation-field application. Environ Model Softw 46:12-20. https://doi.org/10. 1016/j.envsoft.2012.05.003

Krembs FJ, Siegrist RL, Crimi ML, Furrer RF, Petri BG (2010) ISCO for groundwater remediation: analysis of field applications and performance. Groundw Monit Remediat 30(4):42-53

Lee J, Liu X, Kitanidis PK, Kim U, Parker J, Bloom A, Lyon R (2012) Cost optimization of DNAPL remediation at dover air force base site. Ground Water Monit Rem 32(2):48-56. https:// doi.org/10.1111/j1745-6592.2011.01382.x

Li J, He L, Lu H, Fan X (2015) Control of stochastic carcinogenic and noncarcinogenic risks in groundwater remediation through an 
integrated optimization design model. Stoch Environ Res Risk Assess 29:2159-2172

Li J, He L, Chen YZ, Song XS, Lu HW (2017) A bilevel groundwater management model with minimization of stochastic health risks at the leader level and remediation cost at the follower level. Stoch Environ Res Risk Assess 31(10):2547-2571

Loaiciga HA, Charbeneau RJ, Everett LG, Fogg GE, Hobbs BF, Rouhani S (1992) Review of ground-water quality monitoring network design. J Hydrol Eng 118(1):11-37. https://doi.org/10. 1061/(ASCE)0733-9429(1992)118:1(11)

McDade JM, McGuire TM, Newell CJ (2005) Analysis of DNAPL source-depletion costs at 36 field sites. Remediation 15(2):9-18

McGuire TM, McDade JM, Newell CJ (2006) Performance of DNAPL source depletion technologies at 59 chlorinated solventimpacted Site. Ground Water Monit Rem 26(1):73-84

Park E, Parker JC (2005) Evaluation of an upscaled model for DNAPL dissolution kinetics in heterogeneous aquifers. Adv Water Resour 28:1280-1291

Parker JC, Falta RW (2008) Comparison of alternative upscaled model formulations for simulating DNAPL source dissolution and biodecay. Adv Water Resour 31:1325-1332

Parker JC, Kim U (2015) An upscaled approach for transport in media with extended tailing due to back-diffusion using analytical and numerical solutions of the advection dispersion equation. J Contam Hydrol 182:157-172. https://doi.org/10.1016/j.jcon hyd.2015.09.008

Parker JC, Park E (2004) Modeling field-scale DNAPL dissolution kinetics in heterogeneous aquifers. Water Resour Res 40:W05109

Parker JC, Kim U, Widdowson M, Kitanidis P, Gentry R (2010a) Effects of model formulation and calibration data on uncertainty in predictions of DNAPL source dissolution rate. Water Resour Res 46:W12517. https://doi.org/10.1029/2010WR009361

Parker JC, Kim U, Kitanidis P, Cardiff M, Liu X (2010b) Stochastic cost optimization of multi-strategy DNAPL site remediation. Groundw Monit Remediat 30(3):65-78. https://doi.org/10.1111/ j1745-6592.2010.001287.x

Parker JC, Kim U, Kitanidis P, Cardiff M, Liu X, Lee J (2011) Practical cost-optimization of characterization and remediation decisions at DNAPL sites with consideration of prediction uncertainty. SERDP Project ER-1611, final report

Parker JC, Kim U, Fortune A, Griepke S, Galligan J, Bonarrigo A (2017) Data analysis and modeling to optimize thermal treatment cost and performance. Groundw Monit Remediat 37:51-66. https://doi.org/10.1111/gwmr.12199

Rao PSC, Jawitz JW (2003) Comment on "Steady state mass transfer from single component dense nonaqueous phase liquids in uniform flow fields" by T.C. Sale and D.B. McWhorter. Water Resour Res 39(3):1068

Reed PM, Minsker BS, Valocchi AJ (2000) Cost-effective long-term groundwater monitoring design using a genetic algorithm and global mass interpolation. Water Resour Res 36(12):3731-3741

Reitsma S, Dai QL (2001) Reaction-enhanced mass transfer and transport from non-aqueous phase liquid source zones. J Contam Hydrol 49:49-66

Reitsma S, Randhawa J (2002) Experimental investigation of manganese dioxide plugging of porous media. In: Proceedings of the third international conference on remediation of chlorinated and recalcitrant compounds, May 20-23, 2002, Monterey, California

Siegrist RL, Crimi M, Munakata-Marr J, Illagasakare T (2006) Reaction and transport processes controlling in situ chemical oxidation of DNAPLs. SERDP CU-1290, final report

Siegrist RL, Petri B, Krembs F, Crimi M, Ko S, Simpkin T, Palaia T (2008) In situ chemical oxidation for remediation of contaminated groundwater: summary proceedings of an ISCO technology practices workshop. ESTCP Project ER-0623, workshop report

Siegrist RL, Simpkin T, Heiderscheidt J, Illangasekare T et al (2010) In situ chemical oxidation for groundwater remediation-site specific engineering and technology application. ESTCP Project ER-062, final report

Snedecor GW, Cochran WG (1967) Statistical methods. Iowa University Press, Ames Iowa

Waldemer RH, Tratnyek PG (2006) Kinetics of contaminant degradation by permanganate. Environ Sci Technol 40:1055-1061

West MR, Kueper BH (2012) Numerical simulation of DNAPL source zone remediation with in situ chemical oxidation (ISCO). Adv Water Resour 44:126-139

West MR, Grant GP, Gerhard JI, Kueper BH (2007) The influence of precipitate formation on the chemical oxidation of TCE DNAPL with potassium permanganate. Adv Water Resour 31:324-338

Yan E, Schwartz F (2000) Kinetics and mechanisms for TCE oxidation by permanganate. Environ Sci Technol 30:2535-2541

Yang M, Annable MD, Jawitz JW (2016) Solute source depletion control of forward and back diffusion through low-permeability zones. J Contam Hydrol 193:54-62

Publisher's Note Springer Nature remains neutral with regard to jurisdictional claims in published maps and institutional affiliations. 